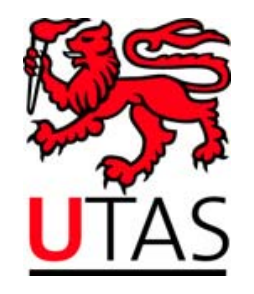

SCHOOL OF ECONOMICS AND FINANCE

Discussion Paper 2013-05

\title{
On Bootstrap Validity for Specification Tests with
} Weak Instruments

Firmin Doko Tchatoka

ISSN 1443-8593

ISBN 978-1-86295-715-2 


\title{
On bootstrap validity for specification tests with weak instruments
}

\author{
Firmin Doko Tchatoka* \\ University of Tasmania
}

July 27,2013

\footnotetext{
* School of Economics and Finance, University of Tasmania, Private Bag 85, Hobart TAS 7001, Phone: +6136226 7226, Fax:+61 36226 7587; e-mail : Firmin.dokotchatoka@utas.edu.au
} 


\begin{abstract}
This paper investigates the asymptotic validity of the bootstrap for Durbin-WuHausman (DWH) specification tests when instrumental variables (IVs) may be arbitrary weak. It is shown that under strong identification, the bootstrap offers a better approximation than the usual asymptotic $\chi^{2}$ distributions. However, the bootstrap provides only a first-order approximation when instruments are weak. These results show unlike the Wald-statistic based on a $k$-class type estimator (Moreira et al., 2009), the bootstrap is valid even for the Wald-type of DWH statistics in the presence of weak instruments.
\end{abstract}

Key words: Specification tests; weak instruments; bootstrap.

JEL classification: C3; C12; C15; C52. 


\section{Introduction}

Specification tests of the type proposed by Durbin (1954), Wu (1973, 1974), and Hausman (1978), henceforth DWH tests, are widely used in applied work to decide whether the ordinary least squares (OLS) or instrumental variables (IV) method is appropriate. Although research on exogeneity testing in linear IV regressions is widespread $^{1}$, most studies in this topic usually consider the case of strong instruments. Recent studies focusing on the behavior of the DWH-type tests document that they never over-rejects the null hypothesis of exogeneity when IVs are weak. However, some of these tests can be overly conservative even in large-sample, and have low power when identification is weak. ${ }^{2}$ Doko Tchatoka and Dufour (2011b) propose a size correction of these tests through the exact Monte carlo test procedure [ Dufour (2006)], which remains valid even when identification is weak and the sample size is small. However, the Monte Carlo test procedure suggested requires the a priori knowledge of the distribution of model disturbance, at least up to an unknown scale factor. But in practice, researchers usually do not know the exact distribution of the errors and implementing the simulated method can be difficult, even infeasible.

This paper aims to relax this distributional assumption by resorting to bootstrap methods. We mainly focus on linear structural models and establish the asymptotic validity of the bootstrap for DWH exogeneity tests, when IVs may be arbitrary weak (weak instruments).

Moreira and Suarez (2009) show in the context of hypotheses specified on struc-

\footnotetext{
${ }^{1}$ See, for example, Durbin (1954), Wu (1973, 1974, 1983a, 1983b), Revankar and Hartley (1973), Farebrother (1976), Hausman (1978), Revankar (1978), Dufour (1979, 1987), Hwang (1980), Kariya and Hodoshima (1980), Hausman and Taylor (1981), Spencer and Berk (1981), Nakamura and Nakamura (1981), Engle (1982), Holly (1982), Reynolds (1982), Smith (1983, 1984), Thurman (1986), Smith and Pesaran (1990), Ruud (1984, 2000), Newey (1985a, 1985b), Wong (1996), Ahn (1997), Baum, Schaffer and Stillman (2003).

${ }^{2}$ See, for examples, Staiger and Stock (1997), Guggenberger (2010), and Doko Tchatoka and Dufour (2011a, 2011b). Staiger and Stock (1997, Section D) show that with weak IVs, the size of Hausman (1978) tests that exploit the residuals from the 2SLS estimation, and that of the Wu (1973) $\mathcal{T}_{3}$ test depends on identification strength through the concentration matrix. Since the concentration matrix cannot be estimated consistently when IVs are weak, Staiger and Stock (1997) conclude that size adjustment of these statistics is infeasible. But Doko Tchatoka and Dufour (2011b) show the size of all DWH-type statistics can be adjusted using the simulated methods; see also Dufour (2006).
} 
tural parameters, that the bootstrap is valid for the score test. This not however the case for Wald-type tests based on the 2SLS or LIML estimators when IVs are weak. We use the $L M$ and Wald interpretation of the DWH staistics in Engle (1982) and Smith (1983) to propose a slight modification of Moreira et al.'s (2009) bootstrap. Our analysis of the bootstrap validity provides some new insights and extensions of Moreira et al.'s (2009). We show that when identification is strong, the bootstrap offers a better approximation than the usual asymptotic $\chi^{2}$ distributions (similar to Moreira et al., 2009). However, the bootstrap provides only a first-order approximation when identification is weak, meaning that the bootstrap is valid even for the Wald-type of the DWH test, despite the lack of identifiability. This contrasts with the bootstrap of the Wald-statistic based on the 2SLS or LIML estimators, which is invalid with weak IVs (Moreira et al., 2009).

The paper is organized as follows. Section 2 formulates the model and assumptions, and presents the statistics studied. Section 3 presents the statistics and provides their Lagrange multiplier or Wald interpretation, following Engle (1982) and Smith (1983). Section 4 details the proposed bootstrap implemented as well as its validity in both strong and weak instrument setups. Conclusions are drawn in Section 5 and the proofs and auxiliary lemmas are presented in the Appendix.

\section{Framework}

We consider the standard linear structural model described by the following equations:

$$
\begin{aligned}
& y_{1}=y_{2} \beta+Z_{1} \gamma+u, \\
& y_{2}=Z_{2} \pi_{2}+Z_{1} \pi_{1}+v_{2}
\end{aligned}
$$

where $y_{1}$ and $y_{2}$ are $n \times 1$ vectors of observations on two endogenous variables, $Z_{1}$ is a $n \times k_{1}$ matrix of included exogenous variables, $Z_{2}$ is a $n \times k_{2}$ matrix instruments, $u=$ $\left(u_{1}, \ldots, u_{n}\right)^{\prime} \in \mathbb{R}^{n}$ is a vector of structural disturbances, $v_{2}=\left[v_{21}, \ldots, v_{2 n}\right]^{\prime} \in \mathbb{R}^{n}$ is 
a vector of reduced form disturbances, $\beta, \gamma \in \mathbb{R}$ are unknown structural parameters, while $\pi_{1} \in \mathbb{R}^{k_{1}}$ and $\pi_{2} \in \mathbb{R}^{k_{2}}$ is the unknown reduced-form coefficient vector. The results in this paper can easily be extended to setups where $y_{2}$ contains more than one regressors. We assume that $Z=\left[Z_{1}: Z_{2}\right]: n \times k$ has full column-rank $k=k_{1}+k_{2}$.

The reduced-forms for $y_{1}$ and $y_{2}$ can be expressed from (2.1)-(2.2) as:

$$
\begin{aligned}
& y_{1}=Z_{1}\left(\pi_{1} \beta+\gamma\right)+Z_{2} \pi_{2} \beta+v_{1} \\
& y_{2}=Z_{1} \pi_{1}+Z_{2} \pi_{2}+v_{2},
\end{aligned}
$$

where $v_{1}=u+v_{2} \beta$. For any random matrix $X$, let $X_{i}$ denote the $i$-th row of $X$, written as column vector. Let $Y=\left[y_{1}: y_{2}\right]$ and define

$$
\mathcal{Q}_{n}=\operatorname{vech}\left(\left(Y_{n}^{\prime}, Z_{n}^{\prime}\right)^{\prime}\left(Y_{n}^{\prime}, Z_{n}^{\prime}\right)\right)=\left(f_{1}\left(Y_{n}^{\prime}, Z_{n}^{\prime}\right), f_{1}\left(Y_{n}^{\prime}, Z_{n}^{\prime}\right), \ldots, f_{l}\left(Y_{n}^{\prime}, Z_{n}^{\prime}\right)\right),
$$

where $f_{i}, i=1, \ldots, l, l=(k+2)(k+3) / 2, k=k_{1}+k_{2}$, are elements of the matrix $\left(Y_{n}^{\prime}, Z_{n}^{\prime}\right)^{\prime}\left(Y_{n}^{\prime}, Z_{n}^{\prime}\right)$. Let $\overline{\mathcal{Q}}_{n}=n^{-1} \sum_{i=1}^{n} \mathcal{Q}_{i}$ denote the empirical mean of the $\mathcal{Q}_{i}$. The following assumptions are made on the behavior of model variables.

Assumption $2.1(a) \mathcal{Q}_{n}$ in (2.4) satisfies: $\mathbb{E}\left[\left\|\mathcal{Q}_{n}\right\|^{s}\right]<\infty$ for some $s \geq 3$, $\limsup _{\|t\| \rightarrow \infty}\left|\mathbb{E}\left[\exp \left(i t^{\prime} Q_{n}\right)\right]\right|<1$; and $(b)$ when the sample size $n$ converges to infinity, the following convergence results hold jointly:

M1. $\quad n^{-1}\left[u: v_{2}\right]^{\prime}\left[u: v_{2}\right] \stackrel{p}{\rightarrow} \Sigma=\left(\begin{array}{cc}\sigma_{u}^{2} & \delta \\ \delta & \sigma_{v_{2}}^{2}\end{array}\right), n^{-1} Z^{\prime} Z \stackrel{p}{\rightarrow} Q_{Z}, n^{-1} Z^{\prime}\left[u: v_{2}\right] \stackrel{p}{\rightarrow} 0$

M2. $\quad n^{-1 / 2} Z^{\prime}\left[u: v_{2}\right] \stackrel{d}{\rightarrow}\left[\psi_{Z u}: \psi_{Z v_{2}}\right]$, where $\psi_{Z u}=\left(\psi_{Z_{1} u}^{\prime}, \psi_{Z_{2} u}^{\prime}\right)^{\prime}: k \times 1$,

$$
\psi_{Z v_{2}}=\left(\psi_{Z_{1} v_{2}}^{\prime}, \psi_{Z_{2} V-2}^{\prime}\right)^{\prime}: k \times 1, \text { and vech }\left(\left[\psi_{Z u}: \psi_{Z v_{2}}\right]\right) \sim N\left(0, \Sigma \otimes Q_{Z}\right) \text {. }
$$

The first moment condition in Assumption 2.1-(a) holds if $\mathbb{E}\left[\left\|\left(Y_{n}^{\prime}, Z_{n}^{\prime}\right)\right\|^{2 s}\right]<\infty$, and the second is the commonly used Cramér's condition [see Bhattacharya and Ghosh 
(1978)]. In Assumption 2.1-(b), M1 is the weak law of large numbers (WLLN) property, where IVs and disturbances are asymptotically uncorrelated, while $M 2$ is the central limit theorem (CLT) property.

From Assumption 2.1, the exogeneity hypothesis of $y_{2}$ can be expressed as:

$$
\mathrm{H}_{0}: \delta=0
$$

We are concerned with the asymptotic validity of the bootstrap for the DWH statistics often used to assess $\mathrm{H}_{0}$, especially when identification is weak. Section 3 presents the DWH statistics and their $L M$ or Wald interpretation.

\section{Lagrange Multiplier and Wald Nature of the Standard DWH Tests}

We consider the statistics $\mathcal{T}_{l}, l=2,3,4$, by $\mathrm{Wu}(1973,1974)$ and three alternative Hausman (1978) type statistics, namely, $\mathcal{H}_{j}, j=1,2$, 3. Let $A_{1}=I_{n}-Z_{1}\left(Z_{1}^{\prime} Z_{1}\right)^{-1} Z_{1}^{\prime}$ and $A_{2}=I_{n}-Z\left(Z^{\prime} Z\right)^{-1} Z^{\prime}$ denote the orthogonal matrices to the spaces spanned by the columns of $Z_{1}$ and $Z$, respectively. The statistics $\mathcal{T}_{l}$ and $\mathcal{H}_{j}$ can be expressed in the unified formulation as:

$$
\begin{aligned}
\mathcal{T}_{l} & =\kappa_{l}(\tilde{\beta}-\hat{\beta})^{2} / \tilde{\omega}_{l}^{2}, \quad l=2,3,4, \\
\mathcal{H}_{j} & =n(\tilde{\beta}-\hat{\beta})^{2} / \hat{\omega}_{j}^{2}, \quad j=1,2,3
\end{aligned}
$$

where $\hat{\beta}=\left(y_{2}^{\prime} A_{1} y_{2}\right)^{-1} y_{2}^{\prime} A_{1} y_{1}$ and $\tilde{\beta}=\left[y_{2}^{\prime}\left(A_{1}-A_{2}\right) y_{2}\right]^{-1} y_{2}^{\prime}\left(A_{1}-A_{2}\right) y_{1}$ are the OLS and IV estimators of $\beta$, respectively, and

$$
\tilde{\omega}_{2}^{2}=\tilde{\sigma}_{2}^{2} \hat{\Delta}, \tilde{\omega}_{3}^{2}=\tilde{\sigma}^{2} \hat{\Delta}, \tilde{\omega}_{4}^{2}=\hat{\sigma}^{2} \hat{\Delta},
$$




$$
\begin{aligned}
\hat{\omega}_{1}^{2} & =\tilde{\sigma}^{2} \hat{\omega}_{i v}^{-1}-\hat{\sigma}^{2} \hat{\omega}_{l s}^{-1}, \hat{\omega}_{2}^{2}=\tilde{\sigma}^{2} \hat{\Delta}, \hat{\omega}_{3}^{2}=\hat{\sigma}^{2} \hat{\Delta} \\
\hat{\Delta} & =\hat{\omega}_{i v}^{-1}-\hat{\omega}_{l s}^{-1}, \hat{\omega}_{i v}=y_{2}^{\prime}\left(A_{1}-A_{2}\right) y_{2} / n, \hat{\omega}_{l s}=y_{2}^{\prime} A_{1} y_{2} / n \\
\tilde{\sigma}^{2} & =\left(y_{1}-y_{2} \tilde{\beta}\right)^{\prime} A_{1}\left(y_{1}-y_{2} \tilde{\beta}\right) / n, \hat{\sigma}^{2}=\left(y_{1}-y_{2} \hat{\beta}\right)^{\prime} A_{1}\left(y_{1}-y_{2} \hat{\beta}\right) / n \\
\tilde{\sigma}_{2}^{2} & =\hat{\sigma}^{2}-(\tilde{\beta}-\hat{\beta})^{2} / \hat{\Delta}, \kappa_{2}=n-2-k_{1}, \kappa_{3}=\kappa_{4}=n-1-k_{1} .
\end{aligned}
$$

Engle (1982) and Smith (1983) show that each statistic in (3.1)-(3.2) has a score or Wald interpretation. The statistics $\mathcal{T}_{2}, \mathcal{T}_{4}$, and $\mathcal{H}_{3}$ are $L M$-type, while $\mathcal{T}_{3}, \mathcal{H}_{1}$, and $\mathcal{H}_{2}$ are quasi-Wald type. ${ }^{3}$ Under $\mathrm{H}_{0}$ and if further Assumption 2.1-(b) holds, all DWH statistics have the usual chi-square asymptotic distributions if model identification is strong. However, $\mathcal{T}_{3}, \mathcal{H}_{1}$, and $\mathcal{H}_{2}$ are overly conservative, and all DWH tests have a low power if IVs are weak, even in large-sample. We question whether a bootstrap technique can improve ${ }^{4}$ the properties of the DWH tests, with or without weak instruments.

\section{Bootstrap Validity for DWH Tests}

Let $\hat{\pi}=\left(Z^{\prime} Z\right)^{-1} Z^{\prime} y_{2}$ denotes the OLS estimator of $\pi=\left(\pi_{1}^{\prime}, \pi_{2}^{\prime}\right)^{\prime}$ in the first stage regression (2.2). Let $\hat{\theta}$ be an estimator of $\beta$ and $\hat{\gamma}$ those of $\gamma$. The bootstrap procedure consists of the following steps:

\footnotetext{
${ }^{3}$ See Smith (1983) for the score interpretation (Eqs. [6] and [9]) and for the quasi-Wald interpretation (Eqs. [7], [8] and [10]). The regression interpretation of these statistics is provided in Hausman (1978), Dufour (1979, 1987), Wooldridge (2009), and Doko Tchatoka and Dufour (2011b).

${ }^{4}$ Due to the $L M$ nature of $\mathcal{T}_{2}, \mathcal{T}_{4}, \mathcal{H}_{3}$, and the result in Moreira et al. (2009), one can project the bootstrap validity for these statistics. But formal proof needs to be established, especially because the primary focus in Moreira et al. (2009) is not exogeneity testing, and there is no discussion in Moreira et al. (2009) related to exogeneity testing. On the other hand, because of the Wald nature of $\mathcal{T}_{1}, \mathcal{T}_{3}, \mathcal{H}_{1}, \mathcal{H}_{2}$, and the bootstrap invalidity result for the Wald-statistic in Moreira et al. (2009), it is not clear whether the bootstrap applies to these statistics. Hence, this note is useful in clarifying these issues. Wong (1996) illustrates through a Monte Carlo experiment that bootstrapping the Hausman (1978) exogeneity test improves both the size and power of the test. Li (2006) extends Wong's (1996) results by allowing for serial correlated errors. Both papers are referenced in the weak instrument literature. However, neither Wong (1996) nor Li (2006) provides a formal proof of the large-sample validity of their bootstrap, even when IVs are strong. Furthermore, the Monte Carlo designs in both papers exclude cases where IVs are poor, because the smallest correlation between each IV and the (possibly) endogenous regressors is set at 0.1 . Although a correlation of 0.1 is not hight, it is not zero or close to either.
} 
1. From observed data, compute $\hat{\pi}$ and $\hat{\theta}$ along with all other things necessary to get the realizations of the statistics $\mathcal{T}_{l}, \mathcal{H}_{j}$, and the residuals from the reduced-form equation $(2.3): \hat{v}_{1}=y_{1}-Z_{1}\left(\hat{\pi}_{1} \hat{\theta}+\hat{\gamma}\right)-Z_{2} \hat{\pi}_{2} \hat{\theta}, \hat{v}_{2}=y_{2}-Z \hat{\pi}$. These residuals are then re-centered by subtracting sample means to yield $\left(\tilde{v}_{1}, \tilde{v}_{2}\right)$.

2. For each bootstrap sample $r=1, \ldots, B$, data are generated as:

$$
y_{1}^{*}=Z_{1}^{*}\left(\hat{\pi}_{1} \hat{\theta}+\hat{\gamma}\right)+Z_{2}^{*} \hat{\pi}_{2} \hat{\beta}+v_{1}^{*}, \quad y_{2}^{*}=Z^{*} \hat{\pi}+v_{2}^{*}
$$

where $Z^{*}=\left[Z_{1}^{*}: Z_{2}^{*}\right]$ and $\left(v_{1}^{*}, v_{2}^{*}\right)$ are drawn independently from the empirical distribution of $Z$ and $\left(\tilde{v}_{1}, \tilde{v}_{2}\right)$. The corresponding bootstrap statistics $\mathcal{T}_{l}^{*^{r}}$ and $\mathcal{H}_{j}^{*^{r}}$ are then computed for each bootstrap sample $r=1, \ldots, B$.

3. The simulated bootstrap $p$-value is obtained as the proportion of bootstrap statistics that are more extreme than the statistics computed from observed data.

4. The bootstrap test rejects the null hypothesis of exogeneity at level $\alpha$ if its p-value is less than $\alpha$.

The above bootstrap steps, though similar to those by Moreira et al. (2009), have a slight difference in the appropriate ${ }^{5}$ estimator of $\hat{\theta}$ to be used; see fn.4 for further details. We now show the asymptotic validity of the bootstrap.

\footnotetext{
${ }^{5}$ Moreira et al. (2009) show that $\hat{\theta}$ must be strongly consistent, i.e.,

$$
\hat{\pi} \stackrel{p}{\rightarrow} \pi \quad \text { and } \quad \hat{\theta} \hat{\pi} \stackrel{p}{\rightarrow} \theta \pi,
$$

for the bootstrap to be valid. In a linear classical setting, the 2SLS and LIML estimators satisfy the sufficient conditions for strong consistency; see Moreira et al (2009, Proposition 4 and fn.3, p.55). The OLS estimator is not qualified for (4.2) if $\delta \neq 0$ (endogeneity). However, under the null hypothesis of exogeneity $(\delta=0)$, as it is the case here, the OLS estimator is consistent and further efficient, no matter how weak the IVs are. For this reason, we prefer OLS to an alternative 2SLS or LIML estimator.
} 


\subsection{High-order approximation with strong instruments}

In this section, we focus on the case where $\pi \neq 0$ is fixed (strong IVs). We can express the bootstrap DHW statistics $\mathcal{T}_{l}^{*}$ and $\mathcal{H}_{j}^{*}$ based on the re-centering residuals as:

$$
\mathcal{T}_{l}^{*}=\left(\sqrt{n} G\left(\overline{\tilde{Q}}_{n}^{*}\right)\right)^{2}, \mathcal{H}_{j}^{*}=\left(\sqrt{n} \tilde{G}\left(\overline{\tilde{Q}}_{n}^{*}\right)\right)^{2} \quad \text { for all } l \text { and } j
$$

where $\overline{\tilde{Q}}_{n}$ and $\overline{\tilde{Q}}_{n}^{*}$ are analogous of $\overline{\mathcal{Q}}_{n}$ in (2.4). $\overline{\tilde{Q}}_{n}$ is based on the sample re-centering residuals and $\overline{\tilde{Q}}_{n}^{*}$ is based on the bootstrap sample residuals. The functions $G($.$) and$ $\tilde{G}($.$) are real-valued Borel measurable functions on \mathbb{R}^{l}$, which satisfy $G\left(\overline{\tilde{Q}}_{n}\right)=0$ and $\tilde{G}\left(\overline{\tilde{Q}}_{n}\right)=0$, due to the re-centered mechanism [similar to Eqs. (A.5)-(A.6) in the Appendix]. Under strong identification, all derivatives of order $s$ and less of the functions $G($.$) and \tilde{G}($.$) are continuous. So, Edgeworth-type expansion { }^{6}$ applies and we have the following theorem.

Theorem 4.1 Bootstrap VAlidity With Strong IVs. Suppose Assumption 2.1 is satisfied. Under $H_{0}$ and if further $\pi \neq 0$ is fixed, we have:

$$
\begin{aligned}
& \left\|\mathbb{P}^{*}\left(\mathcal{T}_{l}^{*} \leq x\right)-\left[\Phi(x)+\sum_{m=1}^{s-2} n^{-m / 2} p_{\mathcal{T}_{l}}^{m}\left(x ; F_{n}, \hat{\beta}, \hat{\pi}\right) \phi(x)\right]^{2}\right\|_{\infty}=o\left(n^{(s-2)}\right), \\
& \left\|\mathbb{P}^{*}\left(\mathcal{H}_{j}^{*} \leq x\right)-\left[\Phi(x)+\sum_{m=1}^{s-2} n^{-m / 2} p_{\mathcal{H}_{j}}^{m}\left(x ; F_{n}, \hat{\beta}, \hat{\pi}\right) \phi(x)\right]^{2}\right\|_{\infty}=o\left(n^{(s-2)}\right) \text { as } n \rightarrow \infty
\end{aligned}
$$

for all $l$ and $j$, where $p_{\mathcal{T}_{l}}^{m}$ and $p_{\mathcal{H}_{j}}^{m}$ are polynomials in $x$ with coefficients depending on $\hat{\beta}, \hat{\pi}$, and the moments of the distribution $F_{n}$ of $\tilde{\mathcal{Q}}_{n}^{*}=\operatorname{vech}\left(\left(\tilde{Y}_{n}^{*^{\prime}}, \tilde{Z}_{n}^{*^{\prime}}\right)^{\prime}\left(\tilde{Y}_{n}^{*^{\prime}}, \tilde{Z}_{n}^{*^{\prime}}\right)\right)$ conditional on $\hat{\mathscr{F}}_{n}=\left\{\left(Y_{1}^{\prime}, Z_{1}^{\prime}\right), \ldots,\left(Y_{n}^{\prime}, Z_{n}^{\prime}\right)\right\} ; \Phi($.$) and \phi($.$) are the cdf and pdf of$ a $N(0,1)$ respectively, while $\|\cdot\|_{\infty}$ stands for the supremum norm.

First, Theorem 4.1 shows that the bootstrap approximates the empirical Edgeworth expansion in Lemma A.1 up to the $o\left(n^{(s-2)}\right)$ order. This is not surprising because the conditional moments of $\mathcal{Q}_{n}^{*}$, given the data $\hat{\mathscr{F}}_{n}$, converge almost surely

\footnotetext{
${ }^{6}$ Such as in Bhattacharya and Ghosh $(1978$, Theorem 2).
} 
to those of $\mathcal{Q}_{n}$ when identification is strong. Second, the results shows that the error based on the bootstrap simulation is of order $n^{-1}$. Therefore, the bootstrap offers a better approximation than the usual asymptotic $\chi^{2}$ distributions, even for the Wald-type versions of the DWH statistics.

\subsection{First-order Validity with Weak Instruments}

High-order approximation of the limiting distributions of the bootstrap as in Theorem 4.1 is not achievable now due to the lack of identification. Indeed, when $\pi_{2}=\pi_{0} / \sqrt{n}$ where $\pi_{0}$ is a $k_{2} \times 1$ constant vector, the functions $G($.$) and \tilde{G}($.$) in (4.3) are non-$ differentiable. $^{7}$ So, the Edgeworth expansion is not applicable. However, we can prove the following theorem on the first-order approximation of the bootstrap when IVs are weak.

Theorem 4.2 Bootstrap VALIDITy WITH WEAK IVs. Suppose Assumptions 2.1 and $H_{0}$ are satisfied. If for some $\delta>0, \mathbb{E}\left(\left\|Z_{i}\right\|^{4+\delta},\left\|v_{i}\right\|^{2+\delta}\right)<\infty$, then we have:

$$
\mathcal{T}_{l}^{*}\left|\hat{\mathscr{F}}_{n} \stackrel{d}{\rightarrow} \chi^{2}(1), \quad \mathcal{H}_{j}^{*}\right| \hat{\mathscr{F}}_{n} \stackrel{d}{\rightarrow} \chi^{2}(1) \text { a.s., for all } l=2,3,4 ; j=1,2,3
$$

when $\pi=\pi_{0} / \sqrt{n}, \pi_{0}$ is a $k \times 1$ constant vector, and $\hat{\mathscr{F}}_{n}=\left\{\left(Y_{1}^{\prime}, Z_{1}^{\prime}\right), \ldots,\left(Y_{n}^{\prime}, Z_{n}^{\prime}\right)\right\}$.

First, since the statistics $\mathcal{T}_{2}, \mathcal{T}_{4}$, and $\mathcal{H}_{3}$ are LM-type and following Moreira et al. (2009), the bootstrap validity for these statistics is predictable. However, the result of the Wald-type of the DWH statistics, $\mathcal{T}_{3}, \mathcal{H}_{1}$, and $\mathcal{H}_{2}$, is less obvious, because the bootstrap is not valid for the Wald-statistic of $\mathrm{H}_{\beta}: \beta=\beta_{0}$ (see Moreira et al., 2009). The key reason behind the bootstrap validity for the Wald-statistic here is that their asymptotic distributions, even when $\delta \neq 0$, do not depend on the unknown nuisance $\operatorname{parameter}^{8} \beta$, with or without weak IVs. Meanwhile, the asymptotic distribution of

\footnotetext{
${ }^{7}$ Note that all DWH statistics depends on $y_{2}^{\prime}\left(A_{1}-A_{2}\right) y_{2} / n$. However, it is straightforward to see that the derivative of the functions $G($.$) and \tilde{G}\left(\right.$.) with respect to $y_{2}^{\prime}\left(A_{1}-A_{2}\right) y_{2} / n$ is not well-defined when $\pi=0$ or does not exist if $\pi=\pi_{0} c_{n}$ for any sequence $c_{n} \downarrow 0$. So, $G($.$) and \tilde{G}($.$) are not smooth$ when IVs are weak, and Edgeworth-type expansion does not apply.

${ }^{8}$ See Wu(1973, Section 3; 1974, Eqs. [3.11]-[3.16]) and Doko Tchatoka and Dufour (2011a, 2011b).
} 
the Wald-statistic of $\mathrm{H}_{\beta}: \beta=\beta_{0}$, based on 2SLS or LIML, depends heavily ${ }^{9}$ on $\beta$ under the weak instrument scenario.

\subsection{Monte Carlo experiment}

We use simulation to examine the size performance of the proposed bootstrap. The DGP is described ${ }^{10}$ by Eqs. (2.1) and (2.2) where the $n$ rows of $\left[u, v_{2}\right]$ are drawn i.i.d. with mean zero and unit variance, and the correlation between $u_{i}$ and $v_{2 i}$ is set at $\rho=0$ under $\mathrm{H}_{0} \cdot Z_{2}$ contains $k_{2}$ instruments, each generated i.i.d $\mathbf{N}(0,1)$ independently of $\left[u, v_{2}\right]$. We vary $k_{2}$ in $\{2,5,20\}$ within the experiment, but the results are consistent with alternative values. The true value of $\beta$ is set at 2 and the reduced-form coefficient $\pi_{2}$ is chosen as $\pi_{2}=\left(\frac{\mu^{2}}{n\left\|Z_{2} \pi_{0}\right\|}\right)^{1 / 2} \pi_{0}$, where $\pi_{0}$ is a vector of ones, $\mu^{2}$ is the concentration parameter characterizing the strength of the IVs. In this experiment, $\mu^{2}$ varies in $\{0,413,1000\} .{ }^{11}$ To account for non-normal errors, $\left[u, v_{2}\right]$ is generated following Kotz et al. (2000):

$$
u_{i}=a+b \varepsilon_{1 i}+c \varepsilon_{1 i}^{2}+d \varepsilon_{1 i}^{3}, \quad v_{2 i}=a+b \varepsilon_{2 i}+c \varepsilon_{2 i}^{2}+d \varepsilon_{2 i}^{3}
$$

where $\left(\varepsilon_{1 i}, \varepsilon_{2 i}\right) \stackrel{\text { i.i.d. }}{\sim} \mathbf{N}\left(0, I_{2}\right)$ for all $i=1, \ldots, n$. We consider two setups: (1) $a=$ $c=d=0$ and $b=1$ (normal errors), and $a=c=0, b=d=1 / \sqrt{22}$ (non-normal errors) such that ${ }^{12}$ Sknew $=0$ and Kurt $\approx 27.72$.

Table 1 presents the results for the standard DWH tests, and Table 2 reports those of the bootstrap tests. The first column of each table contains the test statistics, the second reports the number of IVs $k_{2}$, while the others present, for each sample size $(n)$

\footnotetext{
${ }^{9}$ See Nelson and Startz (1990); Staiger and Stock (1997); Dufour (1997, 2003); Wang and Zivot (1998), among others.

${ }^{10}$ There is no exogenous $Z_{1}$ in the simulations but the results do not alter when such exogenous IVs are included.

${ }^{11}$ Following Hansen et al. (2008), $\mu^{2}=0$ is a design of complete non-identification, $\mu^{2}=413$ designs weak identification, and $\mu^{2}=1000$ is for strong identification.

${ }^{12}$ We run the simulations with alternative values of (Skew, Kurt) and the results are qualitatively similar.
} 
and the IV strength $\left(\mu^{2}\right)$, the empirical rejections of the tests. The bootstrap rejection probability is estimated using 10,000 pseudo-sample sets, each of size $n$ varying in $\{50,100,200,500,1000\}$. The nominal level for both the standard and bootstrap tests is $5 \%$. It is clear from Table 1 that the standard Wald-type of the DWH tests, namely, $\mathcal{T}_{3}, \mathcal{H}_{1}$, and $\mathcal{H}_{2}$, are highly conservative with weak IVs (see columns $\mu^{2}=0$ and $\left.\mu^{2}=413\right)$. The rejection frequencies of the $L M$-type tests $-\mathcal{T}_{2}, \mathcal{T}_{4}$, and $\mathcal{H}_{3}-$ are close to the nominal level of $5 \%$ even when IVs are weak. These results are similar for normal and non-normal errors. Meanwhile, Table 2 shows clearly that the bootstrap method improves the size of the tests, especially for the Wald-type of the DWH tests. As seen, even the rejection frequencies of $\mathcal{T}_{3}, \mathcal{H}_{1}$, and $\mathcal{H}_{2}$ are very close to the nominal level, no matter how weak the IVs are, with or without normal errors, even with relatively small-sample sizes.

\section{Conclusion}

This paper considers the standard linear IV models and investigates the asymptotic validity of the bootstrap for the standard DWH exogeneity tests. We propose a slight modification of Moreira et al.'s (2009) bootstrap, which provides some new insights and extensions of earlier results. When identification is strong, we show that the bootstrap offers a better approximation of the distributions of the statistics than the usual asymptotic $\chi^{2}$ distributions. However, it provides only a first-order approximation when instruments are weak. Unlike the Wald-statistic based on the 2SLS estimator (see Moreira et al., 2009), ours results show that the bootstrap is valid even for the Wald-type of the DWH statistics. This is mainly because even when identification is weak, the asymptotic distributions of all DWH statistics, including the Wald-type ones, do not depend on the unknown structural parameters, while those of the Wald-statistic based on 2SLS or LIML estimator do. 


\section{Acknowledgments}

We are grateful to Professor Richard J. Smith, Manaing Editor of The Econometrics Journal, for his constructive comments and suggestions. We would also like to thank Professors Jean-Marie Dufour, Mardi Dungey and Lynda Khalaf for several useful comments. 
Table 1. Rejection frequencies (in \%) of the standard DWH tests

\begin{tabular}{|c|c|c|c|c|c|c|c|c|c|c|c|c|c|c|c|c|}
\hline \multicolumn{17}{|c|}{ Normal errors } \\
\hline \multirow[b]{2}{*}{ Statistics } & \multirow[b]{2}{*}{$k_{2} \downarrow \mu^{2} \rightarrow$} & \multicolumn{3}{|c|}{$n=50$} & \multicolumn{3}{|c|}{$n=100$} & \multicolumn{3}{|c|}{$n=200$} & \multicolumn{3}{|c|}{$n=500$} & \multicolumn{3}{|c|}{$n=1000$} \\
\hline & & 0 & 413 & 1000 & 0 & 413 & 1000 & 0 & 413 & 1000 & 0 & 413 & 1000 & 0 & 413 & 1000 \\
\hline $\mathcal{T}_{2}$ & 2 & 6.3 & 6.4 & 7.1 & 5.7 & 5.2 & 5.7 & 6.0 & 5.6 & 5.7 & 6.2 & 5.6 & 5.6 & 6.1 & 5.5 & 5.6 \\
\hline $\mathcal{T}_{2}$ & 2 & 5.6 & 5.4 & 5.0 & 5.1 & 5.1 & 5.4 & 4.9 & 5.2 & 5.0 & 4.9 & 5.2 & 5.0 & 4.9 & 4.7 & 4.9 \\
\hline $\mathcal{T}_{3}$ & 2 & 0.1 & 4.0 & 4.2 & 0.0 & 3.7 & 4.6 & 0.0 & 3.7 & 4.2 & 0.1 & 2.9 & 3.8 & 0.0 & 2.0 & 3.6 \\
\hline $\mathcal{T}_{4}$ & 2 & 4.7 & 4.7 & 4.3 & 4.8 & 4.7 & 4.9 & 4.7 & 5.0 & 4.8 & 4.9 & 5.1 & 4.9 & 4.9 & 4.7 & 4.9 \\
\hline $\mathcal{H}_{1}$ & 2 & 0.1 & 3.5 & 3.6 & 0.0 & 3.4 & 4.2 & 0.0 & 3.6 & 4.0 & 0.1 & 2.8 & 3.7 & 0.0 & 1.9 & 3.6 \\
\hline $\mathcal{H}_{2}$ & 2 & 0.1 & 4.2 & 4.3 & 0.0 & 3.8 & 4.7 & 0.0 & 3.8 & 4.3 & 0.1 & 2.9 & 3.8 & 0.0 & 2.0 & 3.6 \\
\hline $\mathcal{H}_{3}$ & 2 & 5.0 & 4.9 & 4.5 & 4.9 & 4.8 & 5.1 & 4.8 & 5.1 & 4.9 & 4.9 & 5.1 & 4.9 & 4.9 & 4.7 & 4.9 \\
\hline $\mathcal{T}_{2}$ & 5 & 5.5 & 5.5 & 5.4 & 5.3 & 5.4 & 5.4 & 4.9 & 5.5 & 5.1 & 4.7 & 5.2 & 5.0 & 5.3 & 4.9 & 5.2 \\
\hline $\mathcal{T}_{3}$ & 5 & 0.3 & 4.5 & 4.7 & 0.3 & 4.9 & 5.0 & 0.3 & 4.6 & 4.8 & 0.4 & 4.2 & 4.6 & 0.3 & 3.9 & 4.5 \\
\hline $\mathcal{T}_{4}$ & 5 & 5.0 & 4.8 & 4.8 & 4.9 & 5.2 & 5.1 & 4.8 & 5.3 & 5.0 & 4.6 & 5.1 & 5.0 & 5.3 & 4.9 & 5.1 \\
\hline $\mathcal{H}_{1}$ & 5 & 0.2 & 3.9 & 4.0 & 0.3 & 4.5 & 4.6 & 0.2 & 4.5 & 4.6 & 0.3 & 4.2 & 4.6 & 0.2 & 3.8 & 4.5 \\
\hline $\mathcal{H}_{2}$ & 5 & 0.3 & 4.8 & 4.9 & 0.3 & 4.9 & 5.1 & 0.3 & 4.7 & 4.8 & 0.4 & 4.2 & 4.7 & 0.3 & 3.9 & 4.5 \\
\hline $\mathcal{H}_{3}$ & 5 & 5.2 & 5.1 & 5.0 & 5.1 & 5.2 & 5.2 & 4.8 & 5.4 & 5.0 & 4.6 & 5.2 & 5.0 & 5.3 & 4.9 & 5.1 \\
\hline $\mathcal{T}_{2}$ & 20 & 5.6 & 5.1 & 5.7 & 5.0 & 5.3 & 5.4 & 5.0 & 5.2 & 5.1 & 4.9 & 4.5 & 5.1 & 5.2 & 4.9 & 5.2 \\
\hline $\mathcal{T}_{3}$ & 20 & 3.3 & 4.5 & 5.1 & 3.0 & 4.9 & 5.0 & 2.8 & 5.0 & 4.8 & 2.9 & 4.3 & 4.9 & 2.8 & 4.6 & 5.1 \\
\hline $\mathcal{T}_{4}$ & 20 & 4.8 & 4.5 & 5.1 & 4.7 & 4.9 & 5.0 & 4.9 & 5.1 & 4.9 & 4.8 & 4.5 & 5.0 & 5.2 & 4.9 & 5.2 \\
\hline $\mathcal{H}_{1}$ & 20 & 2.7 & 3.9 & 4.4 & 2.7 & 4.6 & 4.6 & 2.7 & 4.8 & 4.6 & 2.8 & 4.2 & 4.9 & 2.8 & 4.5 & 5.1 \\
\hline $\mathcal{H}_{2}$ & 20 & 3.5 & 4.6 & 5.3 & 3.1 & 5.0 & 5.1 & 2.8 & 5.0 & 4.9 & 2.9 & 4.3 & 5.0 & 2.9 & 4.6 & 5.1 \\
\hline $\mathcal{H}_{3}$ & 20 & 5.0 & 4.7 & 5.3 & 4.8 & 5.1 & 5.2 & 4.9 & 5.2 & 5.0 & 4.9 & 4.5 & 5.0 & 5.2 & 4.9 & 5.2 \\
\hline \multicolumn{17}{|c|}{ Non-normal errors } \\
\hline \multirow[b]{2}{*}{ Statistics } & \multirow[b]{2}{*}{$k_{2} \downarrow \mu^{2} \rightarrow$} & \multicolumn{3}{|c|}{$n=50$} & \multicolumn{3}{|c|}{$n=100$} & \multicolumn{3}{|c|}{$n=200$} & \multicolumn{3}{|c|}{$n=500$} & \multicolumn{3}{|c|}{$n=1000$} \\
\hline & & 0 & 413 & 1000 & 0 & 413 & 1000 & 0 & 413 & 1000 & 0 & 413 & 1000 & 0 & 413 & 1000 \\
\hline $\mathcal{T}_{2}$ & 2 & 5.0 & 6.3 & 6.1 & 5.2 & 5.0 & 5.6 & 4.8 & 5.0 & 5.0 & 5.0 & 5.0 & 4.7 & 4.9 & 4.9 & 5.4 \\
\hline $\mathcal{T}_{3}$ & 2 & 0.0 & 5.0 & 5.4 & 0.0 & 3.8 & 5.0 & 0.0 & 3.4 & 4.3 & 0.1 & 2.8 & 3.8 & 0.0 & 2.2 & 4.0 \\
\hline $\mathcal{T}_{4}$ & 2 & 4.2 & 5.6 & 5.6 & 4.8 & 4.7 & 5.4 & 4.6 & 4.9 & 4.8 & 5.0 & 5.0 & 4.6 & 4.9 & 4.9 & 5.4 \\
\hline $\mathcal{H}_{1}$ & 2 & 0.0 & 4.4 & 4.8 & 0.0 & 3.5 & 4.7 & 0.0 & 3.3 & 4.1 & 0.0 & 2.8 & 3.7 & 0.0 & 2.1 & 3.9 \\
\hline $\mathcal{H}_{2}$ & 2 & 0.0 & 5.2 & 5.6 & 0.0 & 3.9 & 5.0 & 0.0 & 3.5 & 4.4 & 0.1 & 2.8 & 3.8 & 0.0 & 2.2 & 4.0 \\
\hline $\mathcal{H}_{3}$ & 2 & 4.5 & 5.9 & 5.7 & 5.0 & 4.9 & 5.5 & 4.7 & 5.0 & 4.9 & 5.0 & 5.0 & 4.6 & 4.9 & 4.9 & 5.4 \\
\hline $\mathcal{T}_{2}$ & 5 & 5.3 & 6.0 & 5.9 & 5.2 & 5.4 & 5.9 & 5.2 & 5.1 & 5.0 & 5.0 & 4.3 & 5.2 & 4.9 & 4.8 & 5.0 \\
\hline $\mathcal{T}_{3}$ & 5 & 0.3 & 5.3 & 5.2 & 0.3 & 4.8 & 5.6 & 0.3 & 4.3 & 4.5 & 0.2 & 3.6 & 4.7 & 0.2 & 3.7 & 4.5 \\
\hline $\mathcal{T}_{4}$ & 5 & 4.6 & 5.5 & 5.3 & 4.8 & 5.2 & 5.7 & 5.0 & 4.8 & 4.9 & 4.9 & 4.3 & 5.2 & 4.9 & 4.8 & 5.0 \\
\hline $\mathcal{H}_{1}$ & 5 & 0.2 & 4.7 & 4.6 & 0.2 & 4.5 & 5.2 & 0.2 & 4.2 & 4.3 & 0.2 & 3.5 & 4.6 & 0.2 & 3.7 & 4.5 \\
\hline $\mathcal{H}_{2}$ & 5 & 0.3 & 5.5 & 5.4 & 0.3 & 4.9 & 5.6 & 0.3 & 4.4 & 4.6 & 0.2 & 3.6 & 4.7 & 0.2 & 3.7 & 4.5 \\
\hline $\mathcal{H}_{3}$ & 5 & 4.8 & 5.6 & 5.4 & 4.9 & 5.2 & 5.8 & 5.1 & 4.9 & 4.9 & 4.9 & 4.3 & 5.2 & 4.9 & 4.8 & 5.0 \\
\hline $\mathcal{T}_{2}$ & 20 & 5.5 & 5.8 & 5.8 & 5.1 & 5.2 & 5.6 & 5.2 & 5.1 & 5.4 & 4.9 & 5.2 & 5.0 & 4.8 & 4.9 & 4.8 \\
\hline $\mathcal{T}_{3}$ & 20 & 3.5 & 5.2 & 5.1 & 2.8 & 4.8 & 5.1 & 3.1 & 4.8 & 5.1 & 2.7 & 4.8 & 4.8 & 2.6 & 4.6 & 4.7 \\
\hline $\mathcal{T}_{4}$ & 20 & 4.9 & 5.2 & 5.2 & 4.8 & 4.9 & 5.2 & 5.0 & 4.9 & 5.1 & 4.8 & 5.1 & 4.9 & 4.8 & 4.9 & 4.8 \\
\hline $\mathcal{H}_{1}$ & 20 & 2.8 & 4.6 & 4.5 & 2.6 & 4.5 & 5.0 & 2.9 & 4.6 & 5.0 & 2.7 & 4.8 & 4.8 & 2.6 & 4.6 & 4.7 \\
\hline $\mathcal{H}_{2}$ & 20 & 3.8 & 5.4 & 5.3 & 2.9 & 4.9 & 5.3 & 3.1 & 4.8 & 5.2 & 2.8 & 4.9 & 4.9 & 2.6 & 4.7 & 4.7 \\
\hline $\mathcal{H}_{3}$ & 20 & 5.1 & 5.5 & 5.3 & 4.9 & 5.0 & 5.3 & 5.1 & 5.0 & 5.2 & 4.9 & 5.1 & 5.0 & 4.8 & 4.9 & 4.8 \\
\hline
\end{tabular}


Table 2. Rejection frequencies (in \%) of the bootstrap DWH tests

\begin{tabular}{|c|c|c|c|c|c|c|c|c|c|c|c|c|c|c|c|c|}
\hline \multicolumn{17}{|c|}{ Normal errors } \\
\hline \multirow[b]{2}{*}{ Statistics } & \multirow[b]{2}{*}{$k_{2} \downarrow \mu^{2} \rightarrow$} & \multicolumn{3}{|c|}{$n=50$} & \multicolumn{3}{|c|}{$n=100$} & \multicolumn{3}{|c|}{$n=200$} & \multicolumn{3}{|c|}{$n=500$} & \multicolumn{3}{|c|}{$n=1000$} \\
\hline & & 0 & 413 & 1000 & 0 & 413 & 1000 & 0 & 413 & 1000 & 0 & 413 & 1000 & 0 & 413 & 1000 \\
\hline $\mathcal{T}_{2}^{*}$ & 2 & 6.3 & 6.4 & 7.1 & 5.7 & 5.2 & 5.7 & 6.0 & 5.6 & 5.7 & 6.2 & 5.6 & 5.6 & 6.1 & 5.5 & 5.6 \\
\hline $\mathcal{T}_{3}^{*}$ & 2 & 6.5 & 6.4 & 7.1 & 5.9 & 5.2 & 5.7 & 6.1 & 5.6 & 5.7 & 6.3 & 5.6 & 5.6 & 6.2 & 5.5 & 5.6 \\
\hline $\mathcal{T}_{4}^{*}$ & 2 & 6.3 & 6.4 & 7.1 & 5.7 & 5.2 & 5.7 & 6.0 & 5.6 & 5.7 & 6.2 & 5.6 & 5.6 & 6.1 & 5.5 & 5.6 \\
\hline $\mathcal{H}_{1}^{*}$ & 2 & 6.5 & 6.4 & 7.1 & 5.9 & 5.2 & 5.7 & 6.1 & 5.6 & 5.7 & 6.3 & 5.6 & 5.6 & 6.2 & 5.5 & 5.6 \\
\hline $\mathcal{H}_{2}^{*}$ & 2 & 6.5 & 6.4 & 7.1 & 5.9 & 5.2 & 5.7 & 6.1 & 5.6 & 5.7 & 6.3 & 5.6 & 5.6 & 6.2 & 5.5 & 5.6 \\
\hline $\mathcal{H}_{3}^{*}$ & 2 & 6.3 & 6.4 & 7.1 & 5.7 & 5.2 & 5.7 & 6.0 & 5.6 & 5.7 & 6.2 & 5.6 & 5.6 & 6.1 & 5.5 & 5.6 \\
\hline $\mathcal{T}_{2}^{*}$ & 5 & 6.8 & 5.9 & 5.9 & 6.6 & 6.0 & 6.7 & 7.2 & 5.6 & 5.4 & 6.2 & 5.8 & 5.6 & 7.6 & 5.8 & 5.1 \\
\hline $\mathcal{T}_{3}^{*}$ & 5 & 6.8 & 5.9 & 5.9 & 6.6 & 6.0 & 6.7 & 7.2 & 5.6 & 5.4 & 6.2 & 5.8 & 5.6 & 7.6 & 5.8 & 5.1 \\
\hline $\mathcal{T}_{4}^{*}$ & 5 & 6.8 & 5.9 & 5.9 & 6.6 & 6.0 & 6.7 & 7.2 & 5.6 & 5.4 & 6.2 & 5.8 & 5.6 & 7.6 & 5.8 & 5.1 \\
\hline $\mathcal{H}_{1}^{*}$ & 5 & 6.8 & 5.9 & 5.9 & 6.6 & 6.0 & 6.7 & 7.2 & 5.6 & 5.4 & 6.2 & 5.8 & 5.6 & 7.6 & 5.8 & 5.1 \\
\hline $\mathcal{H}_{2}^{*}$ & 5 & 6.8 & 5.9 & 5.9 & 6.6 & 6.0 & 6.7 & 7.2 & 5.6 & 5.4 & 6.2 & 5.8 & 5.6 & 7.6 & 5.8 & 5.1 \\
\hline $\mathcal{H}_{3}^{*}$ & 5 & 6.8 & 5.9 & 5.9 & 6.6 & 6.0 & 6.7 & 7.2 & 5.6 & 5.4 & 6.2 & 5.8 & 5.6 & 7.6 & 5.8 & 5.1 \\
\hline $\mathcal{T}_{2}^{*}$ & 20 & 6.0 & 5.9 & 6.1 & 7.1 & 6.3 & 6.7 & 6.6 & 6.3 & 6.3 & 6.7 & 5.2 & 5.1 & 7.1 & 5.8 & 5.5 \\
\hline $\mathcal{T}_{3}^{*}$ & 20 & 6.0 & 5.9 & 6.1 & 7.1 & 6.3 & 6.7 & 6.6 & 6.3 & 6.3 & 6.7 & 5.2 & 5.1 & 7.1 & 5.8 & 5.5 \\
\hline $\mathcal{T}_{4}^{*}$ & 20 & 6.0 & 5.9 & 6.1 & 7.1 & 6.3 & 6.7 & 6.6 & 6.3 & 6.3 & 6.7 & 5.2 & 5.1 & 7.1 & 5.8 & 5.5 \\
\hline $\mathcal{H}_{1}^{*}$ & 20 & 6.0 & 5.9 & 6.1 & 7.1 & 6.3 & 6.7 & 6.6 & 6.3 & 6.3 & 6.7 & 5.2 & 5.1 & 7.1 & 5.8 & 5.5 \\
\hline $\mathcal{H}_{2}^{*}$ & 20 & 6.0 & 5.9 & 6.1 & 7.1 & 6.3 & 6.7 & 6.6 & 6.3 & 6.3 & 6.7 & 5.2 & 5.1 & 7.1 & 5.8 & 5.5 \\
\hline $\mathcal{H}_{3}^{*}$ & 20 & 6.0 & 5.9 & 6.1 & 7.1 & 6.3 & 6.7 & 6.6 & 6.3 & 6.3 & 6.7 & 5.2 & 5.1 & 7.1 & 5.8 & 5.5 \\
\hline \multicolumn{17}{|c|}{ Non-normal errors } \\
\hline \multirow[b]{2}{*}{ Statistics } & \multirow[b]{2}{*}{$k_{2} \downarrow \mu^{2} \rightarrow$} & \multicolumn{3}{|c|}{$n=50$} & \multicolumn{3}{|c|}{$n=100$} & \multicolumn{3}{|c|}{$n=200$} & \multicolumn{3}{|c|}{$n=500$} & \multicolumn{3}{|c|}{$n=1000$} \\
\hline & & 0 & 413 & 1000 & 0 & 413 & 1000 & 0 & 413 & 1000 & 0 & 413 & 1000 & 0 & 413 & 1000 \\
\hline $\mathcal{T}_{2}^{*}$ & 2 & 6.4 & 5.8 & 6.4 & 6.0 & 5.7 & 6.0 & 5.7 & 5.2 & 5.2 & 5.5 & 5.3 & 5.8 & 5.5 & 5.0 & 5.2 \\
\hline $\mathcal{T}_{3}^{*}$ & 2 & 6.4 & 5.8 & 6.4 & 5.9 & 5.7 & 6.0 & 5.9 & 5.2 & 5.2 & 5.7 & 5.3 & 5.8 & 5.7 & 5.0 & 5.2 \\
\hline $\mathcal{T}_{4}^{*}$ & 2 & 6.4 & 5.8 & 6.4 & 6.0 & 5.7 & 6.0 & 5.7 & 5.2 & 5.2 & 5.5 & 5.3 & 5.8 & 5.5 & 5.0 & 5.2 \\
\hline $\mathcal{H}_{1}^{*}$ & 2 & 6.4 & 5.8 & 6.4 & 5.9 & 5.7 & 6.0 & 5.9 & 5.2 & 5.2 & 5.7 & 5.3 & 5.8 & 5.7 & 5.0 & 5.2 \\
\hline $\mathcal{H}_{2}^{*}$ & 2 & 6.4 & 5.8 & 6.4 & 5.9 & 5.7 & 6.0 & 5.9 & 5.2 & 5.2 & 5.7 & 5.3 & 5.8 & 5.7 & 5.0 & 5.2 \\
\hline $\mathcal{H}_{3}^{*}$ & 2 & 6.4 & 5.8 & 6.4 & 6.0 & 5.7 & 6.0 & 5.7 & 5.2 & 5.2 & 5.5 & 5.3 & 5.8 & 5.5 & 5.0 & 5.2 \\
\hline $\mathcal{T}_{2}^{*}$ & 5 & 6.9 & 6.6 & 5.9 & 6.0 & 5.3 & 6.0 & 6.3 & 5.3 & 5.5 & 6.7 & 5.8 & 5.1 & 6.9 & 5.5 & 5.4 \\
\hline $\mathcal{T}_{3}^{*}$ & 5 & 6.9 & 6.6 & 5.9 & 6.0 & 5.3 & 6.0 & 6.3 & 5.3 & 5.5 & 6.7 & 5.8 & 5.1 & 6.9 & 5.5 & 5.4 \\
\hline $\mathcal{T}_{4}^{*}$ & 5 & 6.9 & 6.6 & 5.9 & 6.0 & 5.3 & 6.0 & 6.3 & 5.3 & 5.5 & 6.7 & 5.8 & 5.1 & 6.9 & 5.5 & 5.4 \\
\hline $\mathcal{H}_{1}^{*}$ & 5 & 6.9 & 6.6 & 5.9 & 6.0 & 5.3 & 6.0 & 6.3 & 5.3 & 5.5 & 6.7 & 5.8 & 5.1 & 6.9 & 5.5 & 5.4 \\
\hline $\mathcal{H}_{2}^{*}$ & 5 & 6.9 & 6.6 & 5.9 & 6.0 & 5.3 & 6.0 & 6.3 & 5.3 & 5.5 & 6.7 & 5.8 & 5.1 & 6.9 & 5.5 & 5.4 \\
\hline $\mathcal{H}_{3}^{*}$ & 5 & 6.9 & 6.6 & 5.9 & 6.0 & 5.3 & 6.0 & 6.3 & 5.3 & 5.5 & 6.7 & 5.8 & 5.1 & 6.9 & 5.5 & 5.4 \\
\hline $\mathcal{T}_{2}^{*}$ & 20 & 6.1 & 6.2 & 6.2 & 6.2 & 6.2 & 6.5 & 6.4 & 5.9 & 6.2 & 7.1 & 5.9 & 5.8 & 6.9 & 5.7 & 5.1 \\
\hline $\mathcal{T}_{3}^{*}$ & 20 & 6.1 & 6.2 & 6.2 & 6.2 & 6.2 & 6.5 & 6.4 & 5.9 & 6.2 & 7.1 & 5.9 & 5.8 & 6.9 & 5.7 & 5.1 \\
\hline $\mathcal{T}_{4}^{*}$ & 20 & 6.1 & 6.2 & 6.2 & 6.2 & 6.2 & 6.5 & 6.4 & 5.9 & 6.2 & 7.1 & 5.9 & 5.8 & 6.9 & 5.7 & 5.1 \\
\hline $\mathcal{H}_{1}^{*}$ & 20 & 6.1 & 6.2 & 6.2 & 6.2 & 6.2 & 6.5 & 6.4 & 5.9 & 6.2 & 7.1 & 5.9 & 5.8 & 6.9 & 5.7 & 5.1 \\
\hline $\mathcal{H}_{2}^{*}$ & 20 & 6.1 & 6.2 & 6.2 & 6.2 & 6.2 & 6.5 & 6.4 & 5.9 & 6.2 & 7.1 & 5.9 & 5.8 & 6.9 & 5.7 & 5.1 \\
\hline $\mathcal{H}_{3}^{*}$ & 20 & 6.1 & 6.2 & 6.2 & 6.2 & 6.2 & 6.5 & 6.4 & 5.9 & 6.2 & 7.1 & 5.9 & 5.8 & 6.9 & 5.7 & 5.1 \\
\hline
\end{tabular}




\section{APPENDIX}

\section{A. Auxiliary Lemmata and Proofs}

This appendix presents some useful auxiliary lemmas and their proofs, as well as the proofs of the main theorems in the text.

\section{A.1. Auxiliary Lemmata}

Lemma A.1 Suppose Assumption 2.1 is satisfied and that $\pi \neq 0$ is fixed. Under $H_{0}$, we have:

$$
\begin{aligned}
\text { (a) } \quad\left\|\mathbb{P}\left(\sqrt{n} \frac{(\tilde{\beta}-\hat{\beta})}{\tilde{\omega}_{l}} \leq x\right)-\left[\Phi(x)+\sum_{m=1}^{s-2} n^{-m / 2} p_{\mathcal{T}_{l}}^{m}(x ; \tilde{F}, \pi) \Phi(x)\right]\right\|_{\infty}=o\left(n^{(s-2) / 2}\right) \\
\left\|\mathbb{P}\left(\sqrt{n} \frac{(\tilde{\beta}-\hat{\beta})}{\hat{\omega}_{j}} \leq x\right)-\left[\Phi(x)+\sum_{m=1}^{s-2} n^{-m / 2} p_{\mathcal{H}_{j}}^{m}(x ; \tilde{F}, \pi) \Phi(x)\right]\right\|_{\infty}=o\left(n^{(s-2) / 2}\right) \\
\text { (b) } \quad\left\|\mathbb{P}\left(\mathcal{T}_{l} \leq x\right)-\left[\Phi(x)+\sum_{m=1}^{s-2} n^{-m / 2} p_{\mathcal{T}_{l}}^{m}(x ; F, \boldsymbol{\pi}) \Phi(x)\right]^{2}\right\|_{\infty}=o\left(n^{(s-2)}\right), \\
\left\|\mathbb{P}\left(\mathcal{H}_{j} \leq x\right)-\left[\Phi(x)+\sum_{m=1}^{s-2} n^{-m / 2} p_{\mathcal{H}_{j}}^{m}\left(x ; \tilde{F}, b_{0}, \boldsymbol{\pi}\right) \Phi(x)\right]^{2}\right\|_{\infty}=o\left(n^{(s-2)}\right)
\end{aligned}
$$

for all $l$ and $j$, where $p_{\mathcal{T}_{l}}^{m}$ and $p_{\mathcal{H}_{j}}^{m}$ are polynomials in $x$ with coefficients depending on moments of the distribution $F$ of $\mathcal{Q}_{n}$ and $\boldsymbol{\pi}$, and $\Phi($.$) is the cdf of a standard normal$ random variable.

Lemma A.2 Suppose Assumption 2.1 is satisfied. If for some $\delta>0$, we have $\mathbb{E}\left(\left\|Z_{i}\right\|^{2+\delta},\left\|v_{i}\right\|^{2+\delta}\right)<\infty$, then $\mathbb{E}^{*}\left(\left|Z_{j i}^{*} v_{m i}^{*}\right|^{2+\delta}\right)$ is bounded a.s. under $H_{0}$, for all $j=1, \ldots, k$ and $m=1,2 ;$ where $Z^{*}$ and $v^{*}=\left[v_{1}^{*}: v_{2}^{*}\right]$ are the bootstrap draws from the empirical distribution of $Z$ and the re-centered residuals $\tilde{v}=\left[\tilde{v}_{1}: \tilde{v}_{2}\right]$.

Corollary A.3 Under the assumptions of Lemma A.2, $\mathbb{E}^{*}\left(\left|Z_{j i}^{*} u_{i}^{*}\right|^{2+\delta}\right)$ is bounded a.s. under $H_{0}$ for all $j=1, \ldots, k$ and $m=1,2 ;$ where $u^{*}=v_{1}^{*}-v_{2}^{*} \beta$. 
Lemma A.4 Suppose Assumption $\mathbf{2 . 1}$ is satisfied. If for some $\delta>0$, $\mathbb{E}\left(\left\|Z_{i}\right\|^{4+\delta},\left\|v_{i}\right\|^{2+\delta}\right)<\infty$, then under $H_{0}$, we have:

$$
\left(\begin{array}{c}
Z^{*} u^{*} / \sqrt{n} \\
Z^{*} v_{2}^{*} / \sqrt{n} \\
\sqrt{n}\left(\frac{W^{*} 1}{n}-\frac{W^{\prime} 1}{n}\right)
\end{array}\right) \mid \hat{\mathscr{F}}_{n} \stackrel{\stackrel{d}{\rightarrow}}{\rightarrow} \mathbf{N}\left[0,\left(\begin{array}{cc}
\operatorname{diag}\left(\sigma_{u}^{2}, \sigma_{v_{2}}\right) \otimes Q_{Z} & 0 \\
0 & \Sigma_{w}
\end{array}\right)\right] \text { a.s. }
$$

where $W=\left(w_{1}, \ldots, w_{n}\right), \quad w_{i}=\operatorname{vech}\left(Z_{i} Z_{i}^{\prime}\right), W^{*}=\left(w_{1}^{*}, \ldots, w_{n}^{*}\right), w_{i}^{*}=$ $\operatorname{vech}\left(Z_{i}^{*} Z_{i}^{*^{\prime}}\right) \in \mathbb{R}^{k(k+1) / 2}, \Sigma_{w}=\operatorname{var}\left(w_{i}\right)$, and $\mathbb{1}$ is a ( $n$ by 1$)$ constant vector of ones, $\hat{\mathscr{F}}_{n}=\left\{\left(Y_{1}^{\prime}, Z_{1}^{\prime}\right), \ldots,\left(Y_{n}^{\prime}, Z_{n}^{\prime}\right)\right\}$.

Lemma A.5 Suppose Assumption $\mathbf{2 . 1}$ is satisfied. If for some $\delta>0$, $\mathbb{E}\left(\left\|Z_{i}\right\|^{4+\delta},\left\|v_{i}\right\|^{2+\delta}\right)<\infty$, then under $H_{0}$, we have:

$$
\frac{\sqrt{n}\left(\tilde{\beta}^{*}-\hat{\beta}^{*}\right)}{\tilde{\omega}_{l}^{*}}\left|\hat{\mathscr{F}}_{n} \stackrel{d}{\rightarrow} \mathbf{N}(0,1), \quad \frac{\sqrt{n}\left(\tilde{\beta}^{*}-\hat{\beta}^{*}\right)}{\hat{\omega}_{j}^{*}}\right| \hat{\mathscr{F}}_{n} \stackrel{d}{\rightarrow} \mathbf{N}(0,1) \quad \text { a.s. }
$$

when $\pi=\pi_{0} / \sqrt{n}, \pi_{0}$ is a ( $k$ by 1 ) constant vector (and $\pi_{0}=0$ is allowed), where $\tilde{\beta}^{*}, \hat{\beta}^{*}, \tilde{\omega}_{l}^{*}, \hat{\omega}_{j}^{*}$ are the bootstrap counterparts of $\tilde{\beta}, \hat{\beta}, \tilde{\omega}_{l}$, and $\hat{\omega}_{j}$ defined in (3.1)-(3.2).

\section{A.2. Proofs}

To shorten the exposition, note that the proofs of Theorem 4.1 and Lemma A.2 are similar to those in Moreira et al. (2009) and are omitted.

Proof of Lemma A.1 First, it is easy to see that $\mathcal{T}_{l}=c_{n_{l}}\left(\sqrt{n} \frac{(\tilde{\beta}-\hat{\beta})}{\tilde{\omega}_{l}}\right)^{2}$ and $\mathcal{H}_{j}=\left(\sqrt{n} \frac{(\tilde{\beta}-\hat{\beta})}{\hat{\omega}_{j}}\right)^{2}$ for all $l$ and $j$, where $c_{n_{l}}=1+o(1)$. Now, we can observe $\sqrt{n} \frac{(\tilde{\beta}-\hat{\beta})}{\tilde{\omega}_{l}}$ and $\sqrt{n} \frac{(\tilde{\beta}-\hat{\beta})}{\hat{\omega}_{j}}$ as:

$$
\begin{aligned}
& \sqrt{n} \frac{(\tilde{\beta}-\hat{\beta})}{\tilde{\omega}_{l}} \\
= & \sqrt{n} \frac{\left(y_{2}^{\prime} y_{2} / n\right)^{-1}\left(y_{2}^{\prime} y_{1} / n\right)-\left[\left(y_{2}^{\prime} Z / n\right)\left(Z^{\prime} Z / n\right)^{-1}\left(Z^{\prime} y_{2} / n\right)\right]^{-1}\left[\left(y_{2}^{\prime} Z / n\right)\left(Z^{\prime} Z / n\right)^{-1}\left(Z^{\prime} y_{1} / n\right)\right.}{\sqrt{\frac{y_{1}^{\prime} M_{y_{2} y_{1}} y_{1}}{n}\left[\left(\frac{y_{2}^{\prime} P_{Z} y_{2}}{n}\right)^{-1}-\left(\frac{y_{2}^{\prime} y_{2}}{n}\right)^{-1}\right]-\left[\left(\frac{y_{2}^{\prime} y_{2}}{n}\right)^{-1}\left(\frac{y_{2}^{\prime} y_{1}}{n}\right)-\left(\frac{y_{2}^{\prime} P_{Z} y_{2}}{n}\right)^{-1}\left(\frac{y_{2}^{\prime} P_{Z} y_{1}}{n}\right)\right]^{2}}} \\
= & \sqrt{n} \frac{\left(y_{2}^{\prime} y_{2} / n\right)^{-1}\left(y_{2}^{\prime} u / n\right)-\left[\left(y_{2}^{\prime} Z / n\right)\left(Z^{\prime} Z / n\right)^{-1}\left(Z^{\prime} y_{2} / n\right)\right]^{-1}\left[\left(y_{2}^{\prime} Z / n\right)\left(Z^{\prime} Z / n\right)^{-1}\left(Z^{\prime} u / n\right)\right.}{\sqrt{\left.\frac{y_{1}^{\prime} M_{y_{2}} y_{1}}{n}\left[\frac{y_{2}^{\prime} P_{Z} y_{2}}{n}\right)^{-1}-\left(\frac{y_{2}^{\prime} y_{2}}{n}\right)^{-1}\right]-\left[\left(\frac{y_{2}^{\prime} y_{2}}{n}\right)^{-1}\left(\frac{y_{2}^{\prime} y_{1}}{n}\right)-\left(\frac{y_{2}^{\prime} P_{Z} y_{2}}{n}\right)^{-1}\left(\frac{y_{2}^{\prime} P_{Z} y_{1}}{n}\right)\right]^{2}}} \\
= & \sqrt{n} G\left(\bar{Q}_{n}\right) \stackrel{\text { under }}{=} \mathrm{H}_{0} \sqrt{n}\left[G\left(\bar{Q}_{n}\right)-G(\mu)\right]
\end{aligned}
$$




$$
\begin{aligned}
& \sqrt{n} \frac{(\tilde{\beta}-\hat{\beta})}{\hat{\omega}_{j}} \\
& =\sqrt{n} \frac{\left(y_{2}^{\prime} y_{2} / n\right)^{-1}\left(y_{2}^{\prime} y_{1} / n\right)-\left[\left(y_{2}^{\prime} Z / n\right)\left(Z^{\prime} Z / n\right)^{-1}\left(Z^{\prime} y_{2} / n\right)\right]^{-1}\left[\left(y_{2}^{\prime} Z / n\right)\left(Z^{\prime} Z / n\right)^{-1}\left(Z^{\prime} y_{1} / n\right)\right.}{\sqrt{\frac{y_{1}^{\prime} M_{y_{2}} y_{1}}{n}\left[\left(\frac{y_{2}^{\prime} P_{Z} y_{2}}{n}\right)^{-1}-\left(\frac{y_{2}^{\prime} y_{2}}{n}\right)^{-1}\right]}} \\
& =\sqrt{n} \frac{\left(y_{2}^{\prime} y_{2} / n\right)^{-1}\left(y_{2}^{\prime} u / n\right)-\left[\left(y_{2}^{\prime} Z / n\right)\left(Z^{\prime} Z / n\right)^{-1}\left(Z^{\prime} y_{2} / n\right)\right]^{-1}\left[\left(y_{2}^{\prime} Z / n\right)\left(Z^{\prime} Z / n\right)^{-1}\left(Z^{\prime} u / n\right)\right.}{\sqrt{\frac{y_{1}^{\prime} M_{y_{2}} y_{1}}{n}\left[\left(\frac{y_{2}^{\prime} P_{Z} y_{2}}{n}\right)^{-1}-\left(\frac{y_{2}^{\prime} y_{2}}{n}\right)^{-1}\right]}} \\
& =\sqrt{n} \tilde{G}\left(\bar{Q}_{n}\right) \stackrel{\text { under }}{=} \mathrm{H}_{0} \sqrt{n}\left[\tilde{G}\left(\bar{Q}_{n}\right)-\tilde{G}(\mu)\right]
\end{aligned}
$$

where $G($.$) and \tilde{G}($.$) are real-valued Borel measurable functions in \mathbb{R}^{l}$ such that $G(\mu)=G\left(\mathbb{E}\left(\mathcal{Q}_{n}\right)\right)=0$ and $\tilde{G}(\mu)=\tilde{G}\left(\mathbb{E}\left[\mathcal{Q}_{n}\right]\right)=0$ under $\mathrm{H}_{0} \cdot{ }^{13}$ Since $\pi \neq 0$ is fixed (strong identification), all derivatives of $G($.$) and \tilde{G}($.$) of order s$ and less are continuous in the neighborhood of $\mu=0$. So, if further Assumption 2.1-(b) holds, then (A.1)-(A.2) follow directly from Bhattacharya and Ghosh (1978, Theorem 2) and (A.3)-(A.4) hold by the definition of $\mathcal{T}_{l}$ and $\mathcal{H}_{j}$.

Proof of Lemma A.4 Let $\left(c^{\prime}, d^{\prime}\right)^{\prime}$ be a nonzero vector with $c=\left(c_{1}^{\prime}, c_{2}^{\prime}\right)^{\prime} \in \mathbb{R}^{2 k}$ and $d \in \mathbb{R}^{k(k+1) / 2}$. Define

$$
X_{n i}=c_{1}^{\prime} Z_{i}^{*} u_{i}^{*} / \sqrt{n}+c_{2}^{\prime} Z_{i}^{*} v_{2 i}^{*} / \sqrt{n}+d^{\prime}\left(w_{i}^{*}-\bar{w}\right) / \sqrt{n}
$$

where $\left[u_{i}^{*}: v_{2 i}^{*}\right]$ is the $i$-th bootstrap draw of the (re-centered) residuals, and $\bar{w}=$ $n^{-1} \sum_{i=1}^{n} w_{i}, w_{i}=\operatorname{vech}\left(Z_{i} Z_{i}^{\prime}\right) \in \mathbb{R}^{k(k+1) / 2}$, and $w_{i}^{*}=\operatorname{vech}\left(Z_{i}^{*} Z_{i}^{*^{\prime}}\right) \in \mathbb{R}^{k(k+1) / 2}$.

We want to use the Cramér-Wold device. For this, it suffices to show $X_{n i}$ satisfies all the conditions of the Liapunov Central Limit Theorem.

1. The first condition is obvious. Indeed, we have $\mathbb{E}^{*}\left(X_{n i}\right)=0$ by the independence between $Z^{*}$ and $\left[u_{i}^{*}: v_{2 i}^{*}\right]$, and the fact that $\mathbb{E}^{*}\left\{\left[u_{i}^{*}: v_{2 i}^{*}\right]\right\}=0$.

2. The second condition is $\mathbb{E}^{*}\left(X_{n i}^{2}\right)<\infty$. Again, by the independence between $Z^{*}$

\footnotetext{
${ }^{13}$ This holds because $\mathbb{E}\left(y_{2}^{\prime} u\right)=0$ under $\mathrm{H}_{0}$ and $\mathbb{E}\left(Z^{\prime} u\right)=0$ by Assumption 2.1-(a).
} 
and $\left[u_{i}^{*}: v_{2 i}^{*}\right]$ and because $u^{*}$ is uncorrelated with $v_{2}^{*}$ under $\mathrm{H}_{0}$, we have

$$
\mathbb{E}^{*}\left(X_{n i}^{2}\right)=n^{-1}\left\{c_{1}^{\prime}\left(\frac{Z^{\prime} \tilde{u} \tilde{u}^{\prime} Z}{n}\right) c_{1}+c_{2}^{\prime}\left(\frac{Z^{\prime} \tilde{v}_{2} \tilde{v}_{2}^{\prime} Z}{n}\right) c_{2}+d^{\prime} \tilde{\Sigma}_{w} d\right\}<\infty \quad \text { a.s. }
$$

where $\tilde{\Sigma}_{w}=n^{-1} \sum_{i=1}^{n}\left(w_{i}-\bar{w}\right)\left(w_{i}-\bar{w}\right)^{\prime}$.

3. To check the final condition of the Liapunov Central Limit Theorem, it requires to show that $\lim _{n \rightarrow \infty} \sum_{i=2}^{n} \mathbb{E}^{*}\left(\left|X_{n i}\right|^{2+\delta}\right)=0$ a.s. for some $\delta>0$. Now, note that

$$
\begin{aligned}
\sum_{i=2}^{n} \mathbb{E}^{*}\left[\left|X_{n i}\right|^{2+\delta}\right] & =n^{-\delta / 2} n^{-1} \sum_{i=2}^{n} \mathbb{E}^{*}\left[\left|c_{1}^{\prime} Z_{i}^{*} u_{i}^{*}+c_{2}^{\prime} Z_{i}^{*} v_{2 i}^{*}+d^{\prime}\left(w_{i}^{*}-\bar{w}\right)\right|^{2+\delta}\right] \\
& \leq C_{1} n^{-\delta / 2} \mathbb{E}^{*}\left[\left|c_{1}^{\prime} Z_{i}^{*} u_{i}^{*}\right|^{2+\delta}+\left|c_{2}^{\prime} Z_{i}^{*} v_{2 i}^{*}\right|^{2+\delta}+\left|d^{\prime}\left(w_{i}^{*}-\bar{w}\right)\right|^{2+\delta}\right] \\
& \leq C_{2} n^{-\delta / 2}\left\{\sum_{j=1}^{k}\left|c_{1 j}\right|^{2+\delta} \mathbb{E}^{*}\left[\left|Z_{j i}^{*} u_{i}^{*}\right|^{2+\delta}\right]+\sum_{j=1}^{k}\left|c_{2 j}\right|^{2+\delta} \mathbb{E}^{*}\left[\left|Z_{j i}^{*} v_{2 i}^{*}\right|^{2+\delta}\right]+\right\} \\
& +C_{2} n^{-\delta / 2}\left\{\sum_{p=1}^{k(k+1) / 2}\left|d_{p}\right|^{2+\delta} \mathbb{E}^{*}\left[\left|w_{p i}^{*}-\left(\frac{1}{n} \sum_{j=1}^{n} w_{j i}\right)\right|^{2+\delta}\right]\right\} \\
& =C_{2} n^{-\delta / 2}\left[A_{1}+A_{2}+A_{3}\right]
\end{aligned}
$$

for large enough constants $C_{1}$ and $C_{2}$. From Lemma A.2 and Corollary A.3, we have $A_{1}=O(1)$ and $A_{2}=O(1)$ a.s. If further $\mathbb{E}\left[\left\|Z_{i}\right\|^{4+\delta}\right]<\infty$, then e have $A_{3}=O(1)$ a.s. Therefore, we get $\lim _{n \rightarrow \infty} \sum_{i=2}^{n} \mathbb{E}^{*}\left[\left|X_{n i}\right|^{2+\delta}\right]=0$ a.s., and the last condition of the Liapunov Central Limit Theorem is satisfied. Lemma A.4 is the Central Limit Theorem property once we realize that $p \lim _{n \rightarrow \infty}\left(\frac{Z^{\prime} \tilde{u} \tilde{u}^{\prime} Z}{n}\right)=$ $\sigma_{u}^{2} Q_{Z}, p \lim _{n \rightarrow \infty}\left(\frac{Z^{\prime} \tilde{v}_{2} \tilde{v}_{2}^{\prime} Z}{n}\right)=\sigma_{v_{2}}^{2} Q_{Z}$, and $p \lim _{n \rightarrow \infty}\left(\tilde{\Sigma}_{w}\right)=\Sigma_{w}$.

Proof of Lemma A.5 First, note that $\mathbb{E}^{*}\left(Z^{*^{\prime}} Z^{*} / n\right)=Z^{\prime} Z / n, \mathbb{E}^{*}\left(Z^{*^{\prime}} u^{*} / n\right)=$ $Z^{\prime} \tilde{u} / n, \mathbb{E}^{*}\left(Z^{*^{\prime}} v_{2}^{*} / n\right)=Z^{\prime} \tilde{v}_{2} / n$, and $\mathbb{E}^{*}\left[\left(u^{*}: v_{2}^{*}\right)^{\prime}\left(u^{*}: v_{2}^{*}\right) / n\right]=\left(\tilde{u}: \tilde{v}_{2}\right)^{\prime}\left(\tilde{u}: \tilde{v}_{2}\right) / n$. So, 
the Markov law of large numbers entails that

$$
\begin{aligned}
& \frac{Z^{*^{\prime}} Z^{*}}{n}-\frac{Z^{\prime} Z}{n}\left|\hat{\mathscr{F}}_{n} \stackrel{a . s .}{\rightarrow} 0, \frac{Z^{*^{\prime}} u^{*}}{n}-\frac{Z^{\prime} \tilde{u}}{n}\right| \hat{\mathscr{F}}_{n} \stackrel{a . s .}{\rightarrow} 0, \frac{Z^{*^{\prime}} v_{2}^{*}}{n}-\frac{Z^{\prime} \tilde{v}_{2}}{n} \mid \hat{\mathscr{F}}_{n} \stackrel{\text { a.s. }}{\rightarrow} 0 \\
& \frac{1}{n}\left(u^{*}: v_{2}^{*}\right)^{\prime}\left(u^{*}: v_{2}^{*}\right)-\frac{1}{n}\left(\tilde{e}: \tilde{v}_{2}\right)^{\prime}\left(\tilde{u}: \tilde{v}_{2}\right) \mid \hat{\mathscr{F}}_{n} \stackrel{\text { a.s. }}{\rightarrow} 0 ; \quad \text { a.s. }
\end{aligned}
$$

Since $Z^{\prime} Z / n \stackrel{p}{\rightarrow} Q_{Z}$, and $Z^{\prime} \tilde{v}_{2} / n \stackrel{p}{\rightarrow} 0$, we have $Z^{\prime} \tilde{u} / n \stackrel{p}{\rightarrow} 0$ and if $\mathrm{H}_{0}$ holds, $(\tilde{u}$ : $\left.\tilde{v}_{2}\right)^{\prime}\left(\tilde{u}: \tilde{v}_{2}\right) / n \stackrel{p}{\rightarrow} \operatorname{diag}\left(\sigma_{u}^{2}, \sigma_{v_{2}}^{2}\right)$. So, it is clear that: $Z^{*^{\prime}} Z^{*} / n \stackrel{\text { a.s. }}{\rightarrow} Q_{Z}, Z^{*^{\prime}} u^{*} / n \stackrel{\text { a.s. }}{\rightarrow} 0$, $Z^{*^{\prime}} v_{2}^{*} / n \stackrel{a . s .}{\rightarrow} 0$, and $\left(u^{*}: v_{2}^{*}\right)^{\prime}\left(u^{*}: v_{2}^{*}\right) / n \stackrel{a . s .}{\rightarrow} \operatorname{diag}\left(\sigma_{u}^{2}, \sigma_{v_{2}}^{2}\right)$ under $\mathrm{H}_{0}$.

Now, from the above results along with Lemma A.4 and the fact that $\pi=c / \sqrt{n}$, we have: $y_{2}^{*^{\prime}} y_{2}^{*} / n=\pi_{0}^{\prime}\left(Z^{*^{\prime}} Z^{*} / n^{2}\right) \boldsymbol{\pi}_{0}+2 \boldsymbol{\pi}_{0}^{\prime} Z^{*^{\prime}} v_{2}^{*} / n^{3 / 2}+v_{2}^{*^{\prime}} v_{2}^{*} / n$ $\hat{\mathscr{F}}_{n} \stackrel{a . s .}{\rightarrow} \sigma_{v_{2}}^{2}$ and $y_{2}^{*^{\prime}} P_{Z^{*}} y_{2}^{*}=\left(y_{2}^{*^{\prime}} Z^{*} / \sqrt{n}\right)\left(Z^{*^{\prime}} Z^{*} / n\right)^{-1}\left(Z^{*^{\prime}} y_{2}^{*} / \sqrt{n}\right) \mid \hat{\mathscr{F}}_{n} \stackrel{d}{\rightarrow}\left(\psi_{Z v_{2}}+\right.$ $\left.Q_{Z} \boldsymbol{\pi}_{0}\right)^{\prime} Q_{Z}^{-1}\left(\psi_{Z v_{2}}+Q_{Z} \boldsymbol{\pi}_{0}\right)$ a.s., where $\psi_{Z v_{2}} \sim \mathbf{N}\left(0, \sigma_{v_{2}}^{2} Q_{Z}\right)$. Therefore, we have $\tilde{\beta}^{*}-\hat{\beta}^{*}=\left(y_{2}^{*^{\prime}} P_{Z^{*}} y_{2}^{*}\right)^{-1}\left(y_{2}^{*^{\prime}} P_{Z^{*}} u^{*}\right)-\left(y_{2}^{*^{\prime}} y_{2}^{*} / n\right)^{-1}\left(y_{2}^{*^{\prime}} u^{*} / n\right)=\left(y_{2}^{*^{\prime}} P_{Z^{*}} y_{2}^{*}\right)^{-1}\left(y_{2}^{*^{\prime}} P_{Z^{*}} u^{*}\right)+$ $o_{p}(1) \mid \hat{\mathscr{F}}_{n} \stackrel{d}{\rightarrow}\left[\left(\psi_{Z v_{2}}+Q_{Z} \boldsymbol{\pi}_{0}\right)^{\prime} Q_{Z}^{-1}\left(\psi_{Z v_{2}}+Q_{Z} \boldsymbol{\pi}_{0}\right)\right]^{-1}\left(\psi_{Z v_{2}}+Q_{Z} \boldsymbol{\pi}_{0}\right)^{\prime} Q_{Z}^{-1} \psi_{Z u}$ a.s. under $\mathrm{H}_{0}$. Similarly, we can show that $\tilde{\omega}_{l}^{*^{2}} / n, \hat{\omega}_{j}^{*^{2}} / n \mid \hat{\mathscr{F}}_{n} \stackrel{a . s}{\rightarrow} \sigma_{u}^{2}\left[\left(\boldsymbol{\pi}_{0} \psi_{Z v_{2}}+Q_{Z}\right)^{\prime} Q_{Z}^{-1}\left(\psi_{Z v_{2}}+\right.\right.$ $\left.\left.Q_{Z} \boldsymbol{\pi}_{0}\right)\right]^{-1}$ a.s. for all $l$ and $j$. Thus we get

$$
\begin{aligned}
\frac{\sqrt{n}\left(\tilde{\beta}^{*}-\hat{\beta}\right)}{\tilde{\omega}_{l}^{*}} \mid \hat{\mathscr{F}}_{n} \stackrel{d}{\rightarrow} \frac{1}{\sigma_{u}}\left[\left(\psi_{Z v_{2}}+Q_{Z} \boldsymbol{\pi}_{0}\right)^{\prime} Q_{Z}^{-1}\left(\psi_{Z v_{2}}+Q_{Z} \boldsymbol{\pi}_{0}\right)\right]^{-1 / 2}\left(\psi_{Z v_{2}}+Q_{Z} \boldsymbol{\pi}_{0}\right)^{\prime} Q_{Z}^{-1} \psi_{Z u} \\
\frac{\sqrt{n}\left(\tilde{\beta}^{*}-\hat{\beta}^{*}\right)}{\hat{\omega}_{j}^{*}} \mid \hat{\mathscr{F}}_{n} \stackrel{d}{\rightarrow} \frac{1}{\sigma_{u}}\left[\left(\psi_{Z v_{2}}+Q_{Z} \boldsymbol{\pi}_{0}\right)^{\prime} Q_{Z}^{-1}\left(\psi_{Z v_{2}}+Q_{Z} \boldsymbol{\pi}_{0}\right)\right]^{-1 / 2}\left(\psi_{Z v_{2}}+Q_{Z} \boldsymbol{\pi}_{0}\right)^{\prime} Q_{Z}^{-1} \psi_{Z u} \quad \text { a.s. }
\end{aligned}
$$

Moreover, $\psi_{Z u}$ and $\psi_{Z v_{2}}$ are independent and jointly normal under $\mathrm{H}_{0}$ (see also Lemma A.4), thus we have $\frac{1}{\sigma_{u}}\left[\left(\psi_{Z v_{2}}+Q_{Z} \boldsymbol{\pi}_{0}\right)^{\prime} Q_{Z}^{-1}\left(\psi_{Z v_{2}}+Q_{Z} \boldsymbol{\pi}_{0}\right)\right]^{-1 / 2}\left(\psi_{Z v_{2}}+Q_{Z} \boldsymbol{\pi}_{0}\right)^{\prime} Q_{Z}^{-1} \psi_{Z u} \mid$ $\psi_{Z v_{2}} \sim \mathbf{N}(0,1)$. Because the conditional distribution of $\frac{1}{\sigma_{u}}\left[\left(\psi_{Z v_{2}}+Q_{Z} \boldsymbol{\pi}_{0}\right)^{\prime} Q_{Z}^{-1}\left(\psi_{Z v_{2}}+\right.\right.$ $\left.\left.Q_{Z} \boldsymbol{\pi}_{0}\right)\right]^{-1 / 2}\left(\psi_{Z v_{2}}+Q_{Z} \boldsymbol{\pi}_{0}\right)^{\prime} Q_{Z}^{-1} \psi_{Z u}$, given $\psi_{Z v_{2}}$, does not depend on $\psi_{Z v_{2}}$, it is equal to the unconditional distribution. It follows that $\frac{\sqrt{n}\left(\tilde{\beta}^{*}-\hat{\beta}\right)}{\tilde{\omega}_{l}^{*}} \mid \hat{\mathscr{F}}_{n} \stackrel{d}{\rightarrow} \mathbf{N}(0,1)$ and $\frac{\sqrt{n}\left(\tilde{\beta}^{*}-\hat{\beta}^{*}\right)}{\hat{\omega}_{j}^{*}} \mid \hat{\mathscr{F}}_{n} \stackrel{d}{\rightarrow} \mathbf{N}(0,1)$ for all $l=2,3,4$ and $j=1,2,3$. 
Proof OF Theorem 4.2 First, recall that $\mathcal{T}_{l}^{*}=\left(\frac{\sqrt{n}\left(\tilde{\beta}^{*}-\hat{\beta}^{*}\right)}{\tilde{\omega}_{l}^{*}}\right)^{2}$ and $\mathcal{H}_{j}^{*}=$ $\left(\frac{\sqrt{n}\left(\tilde{\beta}^{*}-\hat{\beta}^{*}\right)}{\hat{\omega}_{j}^{*}}\right)^{2}$. By Lemma A.5, we have $\frac{\sqrt{n}\left(\tilde{\beta}^{*}-\hat{\beta}^{*}\right)}{\tilde{\omega}_{l}^{*}} \mid \hat{\mathscr{F}}_{n} \stackrel{d}{\rightarrow} \mathbf{N}(0,1)$ and $\frac{\sqrt{n}\left(\tilde{\beta}^{*}-\hat{\beta}^{*}\right)}{\hat{\omega}_{j}^{*}} \mid$ $\hat{\mathscr{F}}_{n} \stackrel{d}{\rightarrow} \mathbf{N}(0,1)$ a.s. It is clear that $\mathcal{T}_{l}^{*} \mid \hat{\mathscr{F}}_{n} \stackrel{d}{\rightarrow}[\mathbf{N}(0,1)]^{2} \equiv \chi^{2}(1)$ and $\mathcal{H}_{j}^{*} \mid \hat{\mathscr{F}}_{n} \stackrel{d}{\rightarrow}$ $[\mathbf{N}(0,1)]^{2} \equiv \chi^{2}(1)$ a.s.

\section{References}

Ahn, S., 1997. Orthogonality tests in linear models. Oxford Bulletin of Economics and Statistics 59, 83-186.

Baum, C., Schaffer, M., Stillman, S., 2003. Instrumental variables and GMM: Estimation and testing. 3(1), 1-30.

Bhattacharya, R. N., Ghosh, J., 1978. On the validity of the formal edgeworth expansion. The Annals of Statistics 6, 434-451.

Doko Tchatoka, F., Dufour, J.-M., 2011a. Exogeneity tests and estimation in IV regressions. Technical report, Department of Economics, McGill University, Canada Montréal, Canada.

Doko Tchatoka, F., Dufour, J.-M., 2011b. On the finite-sample theory of exogeneity tests with possibly non-gaussian errors and weak identification. Technical report, Department of Economics, McGill University, Canada Montréal, Canada.

Dufour, J.-M., 1979. Methods for Specification Errors Analysis with Macroeconomic Applications PhD thesis University of Chicago. $257+$ XIV pages.

Dufour, J.-M., 1987. Linear Wald methods for inference on covariances and weak exogeneity tests in structural equations. In: I. B. MacNeill, G. J. Umphrey, eds, Advances in the Statistical Sciences: Festschrift in Honour of Professor V.M. 
Joshi's 70th Birthday. Volume III, Time Series and Econometric Modelling. D. Reidel, Dordrecht, The Netherlands, pp. 317-338.

Dufour, J.-M., 1997. Some impossibility theorems in econometrics, with applications to structural and dynamic models. Econometrica 65, 1365-1389.

Dufour, J.-M., 2003. Identification, weak instruments and statistical inference in econometrics. Canadian Journal of Economics 36(4), 767-808.

Dufour, J.-M., 2006. Monte carlo tests with nuisance parameters: A general approach to finite-sample inference and nonstandard asymptotics in econometrics. Journal of Econometrics 138, 2649-2661.

Durbin, J., 1954. Errors in variables. Review of the International Statistical Institute $22,23-32$.

Engle, R. F., 1982. A general approach to Lagrange multiplier diagnostics. Journal of Econometrics 20, 83-104.

Farebrother, R. W., 1976. A remark on the Wu test. Econometrica 44, 475-477.

Guggenberger, P., 2010. The impact of a Hausman pretest on the size of the hypothesis tests. Econometric Theory 156, 337-343.

Hansen, C., Hausman, J., Newey, W., 2008. Estimation with many instrumental variables. Journal of Business and Economic Statistics 26(4), 398-422.

Hausman, J., 1978. Specification tests in econometrics. Econometrica 46, 1251-1272.

Hausman, J., Taylor, W. E., 1981. A generalized specification test. Economics Letters 8, 239-245.

Holly, A., 1982. A remark on Hausman's test. Econometrica 50, 749-759.

Hwang, H.-S., 1980. Test of independence between a subset of stochastic regressors and disturbances. International Economic Review 21, 749-760. 
Kariya, T. , Hodoshima, H. , 1980. Finite-sample properties of the tests for independence in structural systems and LRT. The Quarterly Journal of Economics $31,45-56$.

Kotz, S., Balakrishnan, N., Johnson, N., 2000. Continuous Multivariate Distributions second edn. John Wiley \& Sons, New York.

Li, J., 2006. The block bootstrap test of hausman's exogeneity in the presence of serial correlation. Economics Letters 91, 76-82.

Moreira, Marcelo J.and Porter, J., Suarez, G., 2009. Bootstrap validity for the score test when instruments may be weak. Journal of Econometrics 149, 52-64.

Nakamura, A., Nakamura, M., 1981. On the relationships among several specification error tests presented by Durbin, Wu and Hausman. Econometrica 49, 1583-1588.

Nelson, C., Startz, R., 1990. The distribution of the instrumental variable estimator and its t-ratio when the instrument is a poor one. The Journal of Business $63,125-140$.

Newey, W. K., 1985a. Generalized method of moments specification testing. Journal of Econometrics 29, 229-256.

Newey, W. K., 1985b. Maximum likelihood specification testing and conditional moment tests. Econometrica 53(5), 1047-1070.

Revankar, N. S., 1978. Asymptotic relative efficiency analysis of certain tests in structural sysytems. International Economic Review 19, 165-179.

Revankar, N. S., Hartley, M. J., 1973. An independence test and conditional unbiased predictions in the context of simultaneous equation systems. International Economic Review 14, 625-631. 
Reynolds, R. A., 1982. Posterior odds for the hypothesis of independence between stochastic regressors and disturbances. International Economic Review 23(2), 479-490.

Ruud, P. A., 1984. Tests of specification in econometrics. Econometric Reviews $3(2), 211-242$.

Ruud, P. A., 2000. An Introduction to Classical Econometric Theory. Oxford University Press, Inc., New York.

Smith, R. J., 1983. On the classical nature of the Wu-Hausman statistics for independence of stochastic regressors and disturbance. Economics Letters 11, 357-364.

Smith, R. J., 1984. A note on likelihood ratio tests for the independence between a subset of stochastic regressors and disturbances. International Economic Review $25,263-269$.

Smith, R. J., Pesaran, M., 1990. A unified approach to estimation and orthogonality tests in linear single-equation econometric models. Journal of Econometrics 44, 41-66.

Spencer, D. E., Berk, K. N., 1981. A limited-information specification test. Econometrica 49, 1079-1085. Erratum, Econometrica, Vol. 50, No. 4 (Jul., 1982), p. 1087.

Staiger, D., Stock, J. H., 1997. Instrumental variables regression with weak instruments. Econometrica 65(3), 557-586.

Thurman, W., 1986. Endogeneity testing in a supply and demand framework. Review of Economics and Statistics 68(4), 638-646.

Wang, J., Zivot, E., 1998. Inference on structural parameters in instrumental variables regression with weak instruments. Econometrica 66(6), 1389-1404. 
Wong, K.-f., 1996. Bootstrapping Hausman's exogeneity test. Economics Letters $53,139-143$.

Wooldridge, J. M., 2009. Introductory Econometrics: A Modern Approach fourth edn. South-Western, USA.

Wu, D.-M., 1973. Alternative tests of independence between stochastic regressors and disturbances. Econometrica 41, 733-750.

Wu, D.-M., 1974. Alternative tests of independence between stochastic regressors and disturbances: Finite sample results. Econometrica 42, 529-546.

Wu, D.-M., 1983a. A remark on a generalized specification test. Economics Letters $11,365-370$.

Wu, D.-M., 1983b. Tests of causality, predeterminedness and exogeneity. International Economic Review 24(3), 547-558. 


\section{School of Economics and Finance Discussion Papers}

2013-05

2013-04

2013-03

2013-02

2013-01

2012-11

2012-10

2012-09

2012-08

2012-07

2012-06

2012-05

2012-04

2012-03

2012-02

2012-01

2011-06

2011-05

2011-04

2011-03

2011-02

2011-01

On Bootstrap Validity for Specification Tests with Weak Instruments, Firmin Doko Tchatoka

Chinese Monetary Expansion and the US Economy, Joaquin L Vespignani and Ronald A Ratti

International Monetary Transmission to the Euro Area: Evidence from the US, Japan and China,

Joaquin L Vespignani and Ronald A Ratti

The impact of jumps and thin trading on realized hedge ratios? Mardi Dungey, Olan T. Henry, Lyudmyla Hvozdyk

Why crude oil prices are high when global activity is weak?, Ronald A Rattia and Joaquin L Vespignani Crude Oil Prices and Liquidity, the BRIC and G3 Countries, Ronald A Ratti and Joaquin L Vespignani Crude Oil Prices: China’s Influence Over 1996-2011, Ronald A Ratti and Joaquin L Vespignani Exchange Rate Risk Exposure and the Value of European Firms, Fabio Parlapiano and Vitali Alexeev

Ranking Systemically Important Financial Institutions, Mardi Dungey, Matteo Luciani and David Veredas

Identification-Robust Inference for Endogeneity Parameters in Linear Structural Models, Firmin Doko Tchatoka and Jean-Marie Dufour

Specification Tests with Weak and Invalid Instruments, Firmin Doko Tchatoka

Liquidity and Crude Oil Prices: China’s Influence Over 1996-2011, Ronald A. Rattia and Joaquin L. Vespignani

On the Validity of Durbin-Wu-Hausman Tests for Assessing Partial Exogeneity Hypotheses with Possibly Weak Instruments, Firmin Doko Tchatoka

Endogenous Crisis Dating and Contagion Using Smooth Transition Structural GARCH, Mardi Dungey, George Milunovich, Susan Thorp and Minxian Yang

\section{Testing for Partial Exogeneity with Weak Identification, Firmin Doko Tchatoka}

On the Correspondence Between Data Revision and Trend-Cycle Decomposition, Mardi Dungey, Jan PAM Jacobs and Jian Tian

Systematic and Liquidity Risk in Subprime-Mortgage Backed Securities, Mardi Dungey, Gerald P. Dwyer and Thomas Flavin

A SVECM Model of the UK Economy and The Term Premium, Mardi Dungey and M. Tugrul Vehbi

Do Contact Matter in the Process of Getting a Job in Cameroon? Firmin Doko Tchatoka and Urbain Thierry Yogo

Subset Hypotheses Testing and Instrument Exclusion in the Linear IV Regression, Firmin Doko Tchatoka

First home Buyers’ Support Schemes in Australia - Results Spreadsheet, Mardi Dungey, Graeme Wells and Sam Thompson

First home Buyers’ Support Schemes in Australia, Mardi Dungey, Graeme Wells and Sam Thompson

Copies of the above mentioned papers and a list of previous years' papers are available from our home site at http://www.utas.edu.au/economics-finance/research/ 